\title{
Macromolecular Serial Crystallography
}

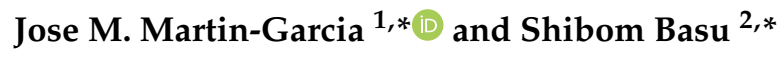 \\ 1 Department of Crystallography and Structural Biology, Institute of Physical Chemistry “Rocasolano" (CSIC), \\ 28006 Madrid, Spain \\ 2 EMBL Grenoble, 71 Avenue des Martyrs, CS 90181, 38042 Grenoble, France \\ * Correspondence: jmmartin@iqfr.csic.es (J.M.M.-G.); shbasu@embl.fr (S.B.)
}

Received: 17 November 2020; Accepted: 24 November 2020; Published: 26 November 2020

Within the structural biology field, X-ray crystallography prevails as the dominant technique to determine the structures of macromolecules, producing, as of November 2020, more than 150,000 structures since its inception (https://www.rcsb.org/). New technologies developed at X-ray free electron lasers (XFELs) and the birth of serial femtosecond crystallography (SFX) have opened up a new era in structural biology. SFX relies on exceptionally bright, micro-focused and ultra-short X-ray beam pulses to probe nano/micrometer sized crystals, delivered in serial fashion at room temperature, resulting in data sets comprised of millions of individual snapshots. As a result, the field is quickly expanding, and allowing structural biologists access to previously restricted scientific areas (e.g., structure dynamics studies). This emerging field has also cultivated new methods for crystallization, and the assessment of nano/microcrystals, sample delivery, and data processing.

In addition to its use at XFELs, the serial crystallography (SX) approach can also be done at synchrotron radiation sources and has gained popularity in the past few years, up to the point that it can now be a viable alternative to the scarce X-ray free electron laser sources. Monochromatic and pink beam experiments have demonstrated the feasibility of serial data collection using micro-crystals at numerous microfocus beamlines at the most powerful synchrotron radiation sources in the world. Upcoming developments in beamline optics, detector technology and synchrotron sources by themselves will enable the use of even smaller micro-crystals $(<1 \mu \mathrm{m})$, the use of larger macromolecules as well as the possibility of conducting mix-and-inject time-resolved studies.

The main goal of this Special Issue on "Macromolecular Serial Crystallography" has been to gather research manuscripts from experts in the field (chemists, biologists, physicists, and structural biologists) to create an international platform to provide rich and reference information on the latest advances and exciting discoveries in the still emerging technology of serial crystallography at XFELs and synchrotron radiation sources. This special issue collects four interesting manuscripts-a preface [1] on serial crystallography, two reviews [2,3], and a research manuscript [4].

The special issue starts with a preface on serial crystallography by John Spence [1], a pioneer of the SFX technique at XFELs, along with Henry Chapman (CFEL, Hamburg, Germany) and Petra Fromme (ASU, USA). Spence's manuscript provides a good overview of some recent work done at XFELs, with a survey of the rapid advances made over the past decade in both sample delivery and data analysis.

The review article by Cheng [2] is a comprehensive review that covers most of the current and future trends on sample delivery methods for SFX at XFELs. The sample delivery has become an active area of research among XFEL and synchrotron sources. The latest developments in this field are aimed at reducing sample consumption and background together with facilitating time-resolved SFX (TR-SFX) experiments. Numerous delivery techniques (and devices) have been emerging since the inception of serial crystallography ten years ago. The pros and cons of those techniques are critically discussed from an industrial user's perspective in Cheng's article. It is important to have opinions from 
industrial users for future technical advances and bring the technique to pharmaceutical industries. One of the major advantages of this technique is the high-throughput over conventional data collection. Serial crystallography allows the user to screen a thousand times more samples at a given beamtime. This feature can encourage industrial users to use serial crystallography as routine if some of the critical points raised by Cheng's article are technically addressed in future.

The review article by Schmidt [3], highlights the most recent advances made in the field of TR-SFX of reactions by rapid mixing experiments, as well as points out the benefits in the field of upcoming high-repetition rate XFELs. This article discusses the pros and cons of doing mixing experiments at synchrotron radiation sources by comparing them with XFELs. A variety of mixing techniques currently available for structure-based enzymology are also discussed.

The research article by Kupitz and Sierra [4] collects the best practices on how to prepare protein samples for a successful beamtime for an SFX experiment at XFELs by addressing some of the most common pitfalls. In science, we all tend to exaggerate the successes and underreport the failures. The authors addressed such failures with various troubleshooting details. Based on their wide experiences, they eloquently discussed proper protein crystal concentration for liquid jet experiments, aiming for mono-dispersity and filtering, crushing big synchrotron crystals, as well as selecting the most appropriate sample delivery methods, among many more. We (the guest editors of this Special Issue), as experienced XFELs users, believe that an article like that of Kupitz and Sierra is urgently needed to expand the user community as it may enable beginners in the field to perform SFX experiments with a higher success rate.

We would like to thank all authors who have contributed with their excellent manuscripts to this Special Issue, the reviewers who provided constructive and helpful feedback on all submissions, and the Editorial Office staff at Crystals for their fast and professional handling of all manuscripts during the submission process and for the help provided throughout.

Funding: Jose M. Martin-Garcia was funded by the Community of Madrid through the "Atracción y Retención de Talento" Grant (Ref: 2019-T1BMD-1552), and Shibom Basu was funded by The European Molecular Biology Laboratory (EMBL).

Conflicts of Interest: The authors declare no conflict of interest.

\section{References}

1. Spence, J.C.H. Serial Crystallography: Preface. Crystals 2020, 10, 135. [CrossRef]

2. Cheng, R. Towards an Optimal Sample Delivery Method for Serial Crystallography at XFEL. Crystals 2020, 10, 215. [CrossRef]

3. Schmidt, M. Reaction Initiation in Enzyme Crystals by Diffusion of Substrate. Crystals 2020, 10, 116. [CrossRef]

4. Kupitz, C.; Sierra, R.G. Preventing Bio-Bloopers and XFEL Follies: Best Practices from your Friendly Instrument Staff. Crystals 2020, 10, 251. [CrossRef]

Publisher's Note: MDPI stays neutral with regard to jurisdictional claims in published maps and institutional affiliations.

(C) 2020 by the authors. Licensee MDPI, Basel, Switzerland. This article is an open access article distributed under the terms and conditions of the Creative Commons Attribution (CC BY) license (http://creativecommons.org/licenses/by/4.0/). 